\title{
Correction to: Restitution metrics in Brugada syndrome: a systematic review and meta-analysis
}

\author{
Gary Tse ${ }^{1,2}$ (1) - Sharen Lee ${ }^{3} \cdot$ Mengqi Gong ${ }^{2} \cdot$ Panagiotis Mililis $^{4} \cdot$ Dimitrios Asvestas $^{4} \cdot$ George Bazoukis $^{4}$.

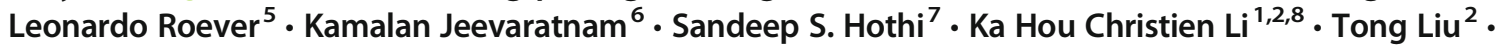 \\ Konstantinos P. Letsas ${ }^{4}$
}

Published online: 15 January 2020

(C) Springer Science+Business Media, LLC, part of Springer Nature 2020

\section{Correction to: Journal of Interventional Cardiac Electrophysiology}

https://doi.org/10.1007/s10840-019-00675-z

The original version of this article unfortunately has a typo error. The name of the author "Kamalan Jeeveratnam" should be presented as "Kamalan Jeevaratnam" as shown above.

The original article has been corrected.

The online version of the original article can be found at https://doi.org/ 10.1007/s10840-019-00675-z

Gary Tse

gary.tse@ doctors.org.uk

$\triangle$ Konstantinos P. Letsas

k.letsas@gmail.com

1 Xiamen Cardiovascular Hospital Affiliated to Xiamen University, Xiamen, Fujian, People's Republic of China

2 Tianjin Key Laboratory of Ionic-Molecular Function of Cardiovascular Disease Department of Cardiology, Tianjin Institute of Cardiology, Second Hospital of Tianjin Medical University, Tianjin 300211, People's Republic of China
3 Laboratory of Cardiovascular Physiology, Li Ka Shing Institute of Health Sciences, Hong Kong, SAR, People's Republic of China

4 Second Department of Cardiology, Laboratory of Cardiac Electrophysiology, Evangelismos General Hospital of Athens, Athens, Greece

5 Department of Clinical Research, Federal University of Uberlândia, Uberlândia, MG, Brazil

6 Faculty of Health and Medical Sciences, University of Surrey, Guildford, UK

7 Heart and Lung Centre, New Cross Hospital, Wolverhampton, UK

8 Faculty of Medicine, Newcastle University, Newcastle, UK 